\title{
Creación de un cuestionario para describir los hábitos de prescripción de antibióticos de odontólogos en Chile.
}

\section{Creation of a survey to describe the antibiotic prescription habits of dentists in Chile.}

\author{
Florencia Hanisch-Cerda ${ }^{{ }^{*}}$, Antonio Quintero-Harvey ${ }^{1}$
}

\begin{abstract}
1. Área Periodoncia, Facultad de Odontología. Universidad de los Andes. Santiago, Chile.
\end{abstract}

* Correspondencia Autor: Florencia Hanisch Cerda | Dirección: Universidad de los Andes. Monseñor Álvaro del Portillo 12.455, Las Condes. Santiago, Chile. | Teléfono: +562 26181372 | E-mail: florenciahanisch@gmail.com

Trabajo recibido el 04/01/2020

Aprobado para su publicación el 11/07/2020

\begin{abstract}
RESUMEN
Antecedentes: El consumo de antibióticos se ha convertido en un problema de salud pública. Aproximadamente un $10 \%$ de los antibióticos son indicados por odontólogos. Algunos países han identificado las prácticas clínicas relacionadas con su indicación por medio de cuestionarios de auto-reporte. En Chile no existe información respecto al uso e indicación de antibióticos de los odontólogos. Objetivo: Diseñar y validar un instrumento para el levantamiento de información sobre la indicación de antibióticos por los odontólogos en Chile. Método: Se diseñó una investigación en dos etapas: 1) Creación de un cuestionario de auto-reporte como instrumento de levantamiento de información. 2) Pilotaje del cuestionario y análisis descriptivo sobre los hábitos de prescripción. Resultados: Se creó un cuestionario de 88 preguntas, organizadas principalmente en matrices. El Content Validity Index fue 0.88 . En el pilotaje se analizaron 418 respuestas. El 51,67\% fueron de la Región Metropolitana, $43 \%$ de odontólogos tiene más de 10 años de experiencia, $50 \%$ declara tener estudios de postítulo, $81 \%$ señaló prescribir antibióticos entre $1 \%$ y $25 \%$ de sus pacientes en un mes de trabajo. Amoxicilina es el antibiótico más indicado. Conclusión: Se valida el instrumento diseñado para levantar información respecto a la indicación de antibióticos por los odontólogos en Chile.
\end{abstract}

\section{PALABRAS CLAVE}

Cuestionario; Antibióticos; Odontólogos; Auto-reporte.

Int. J. Inter. Dent Vol. 13(2); 67-70, 2020.

\begin{abstract}
Background: Antibiotic consumption has become a public health problem. Dentists prescribe approximately $10 \%$ of the antibiotics. Some countries have identified clinical practices related to their prescription through self-report questionnaires. In Chile, there is no information regarding the prescription of antibiotics by dentists. Aim: Design and validate an instrument that allows for collecting information about antibiotic prescription by dentists in Chile. Method: An investigation was designed in two stages: 1) Creation of a self-report questionnaire as an information-gathering instrument. 2) A pilot study of the questionnaire to perform a descriptive analysis of prescription habits. Results: A questionnaire of 88 questions was created, organized mainly in matrices. The Content Validity Index was 0.88. During the pilot, 418 responses were analyzed. $51.67 \%$ of the dentists were from the Metropolitan Region, $43 \%$ had more than ten years of experience, $50 \%$ declared having postgraduate studies, $81 \%$ indicated that they prescribed antibiotics to between $1 \%$ and $25 \%$ of their patients in one month of work. Amoxicillin is the most prescribed antibiotic. Conclusion: The instrument designed was validated to evaluate the antibiotic prescription habits by dentists in Chile.

KEY WORDS

Questionnaire; Antibiotics; Dentists; Self-report.

Int. J. Inter. Dent Vol. 13(2); 67-70, 2020.
\end{abstract}

\section{INTRODUCCIÓN}

El acceso a medicamentos, de calidad, con un uso racional y sobre fundamentos científico-técnicos, permite la obtención de importantes beneficios en materia de prevención, diagnóstico y tratamiento de las enfermedades y sus síntomas ${ }^{(1)}$

En Chile, la Política Nacional de Medicamentos, establece la necesidad de implementar estrategias de uso racional de medicamentos en todos los niveles, propiciando la obtención de un uso adecuado y eficiente de estos ${ }^{(1,2)}$.

Según estadísticas de la Organización Mundial de la Salud (OMS), más de un $50 \%$ de todos los medicamentos a nivel mundial se recetan, dispensan o venden de manera inadecuada con un impacto financiero de aproximadamente USD 5.000 millones en Estados Unidos y USD 10.000 millones en Europa $a^{(2,3)}$.

Si bien la utilidad de los antibióticos es indiscutible, su uso indiscriminado puede llevar a un aumento en la resistencia microbiana adquirida a los antibióticos. Para la OMS, la resistencia microbiana corresponde a uno de los grandes desafíos de salud para el siglo XXI ${ }^{(4)}$.

Se considera que entre un 7 y un $10 \%$ de los antibióticos son indicados por los odontólogos ${ }^{(5-7)}$ y sus hábitos de prescripción han sido evaluados, utilizando cuestionarios de auto-reportes, en países como Reino Unido, Estados Unidos, Noruega, Brasil y Alemania, entre otros ${ }^{(6-13)}$. En Chile, a pesar 
de ser los antibióticos uno de los grupos de medicamentos de mayor venta, con una distribución masiva y con una alta penetración en nuestra población, no tenemos información en relación a su prescripción e indicaciones de uso, tanto terapéutico como profiláctico, entre los odontólogos que ejercen la profesión en nuestro país.

En este estudio se tiene como objetivo principal, el diseño y validación de un cuestionario de auto-reporte como instrumento de levantamiento de información sobre prescripción de antibióticos por los odontólogos en Chile, de manera terapéutica y profiláctica, en distintas situaciones clínicas de los pacientes.

\section{METODOLOGÍA}

Se realizó un estudio en dos etapas: La primera consistió en la creación del cuestionario de auto-reporte y la segunda en la aplicación del instrumento a un grupo de odontólogos, del cual se obtuvieron y cuantificaron los datos.

\section{Construcción del cuestionario de auto-reporte}

Para la construcción del cuestionario se consideraron diferentes fuentes de referencia para rescatar tópicos relevantes de ser incluidos. Fueron utilizados investigaciones anteriores de otros grupos de investigadores, entrevistas a informantes claves y opinión de expertos ${ }^{(14)}$ (ver Figura 1). Para todas las fuentes de referencia se consideró la diversidad como un valor. Así, las investigaciones previas fueron de diferentes países y se utilizaron tanto investigaciones sobre prescripción de antibióticos como de diseño de instrumentos de levantamiento de información ${ }^{(8-12)}$. Los expertos a quienes se les solicitó la opinión pertenecieron a diversas áreas tanto de salud como de ciencias sociales ${ }^{(15)}$ y los informantes claves fueron odontólogos de diferentes especialidades.

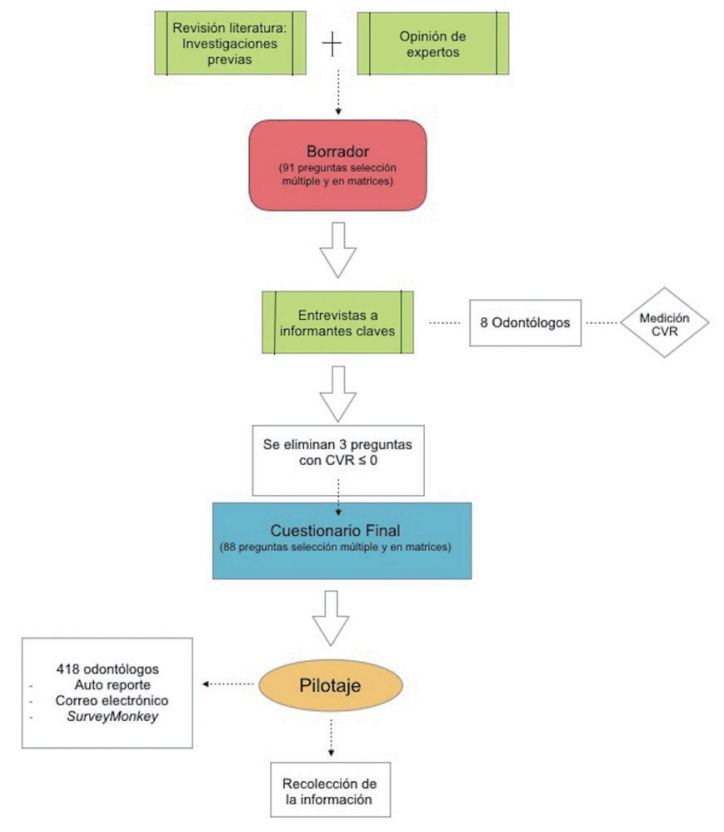

Figura 1. Construcción del cuestionario.

El borrador inicial fue elaborado por los autores basándose en estudios similares realizados en otros países, además de la participación de 3 expertos, los cuales brindaron ayuda para la creación de las preguntas y su estructuración. Participaron como expertos una psicóloga economista, con experiencia en la construcción de este tipo de instrumentos de medición, quien participó en la estructura, forma y redacción de las preguntas, un químico farmacéutico, docente universitario de farmacología, que colaboró con los antibióticos a preguntar y dosis de estos y un cirujano maxilofacial que asesoró desde el punto de vista clínico, es decir, sobre qué condiciones odontológicas y médicas consultar. Su participación se realizó como un proceso iterativo donde se les solicitó que complementaran y corrigieran el borrador creado(14). Las reuniones fueron presenciales y con cada uno de los expertos por separado y la información fue recopilada por F.H. Los aportes y comentarios de los expertos llevaron a modificar sustancialmente las preguntas del cuestionario y permitió la creación del borrador final. Luego se solicitó la participación de informantes claves para poder medir el grado de entendimiento del cuestionario. Se calculó la validez aparente y de contenido por medio del Content Validity Ratio (CVR) y el Content Validity
Index (CVI) ${ }^{(16,17)}$. Para esto se les solicitó que categorizaran cada pregunta del cuestionario como "esencial", "útil, pero no esencial" o "innecesario", calculando así, el CVR para cada pregunta. La validez aparente es la determinación del grado en el que las preguntas parecen medir lo que se proponen. Para esto se solicitó a los informantes que pusieran su opinión, sugerencias o comentarios en cada pregunta. De esta manera fueron modificados la redacción u orientación de algunos ítems. La validez de contenido se refiere al grado en que los elementos de un instrumento son relevantes y representativos de lo que se quiere evaluar ${ }^{(15,18)}$ y fue medido mediante el CVR y CVI.

Cada pregunta del cuestionario fue considerada una variable independiente ${ }^{(18)}$ y fueron contempladas las siguientes categorías $^{(8-12)}$ : aspectos demográficos, aspectos generales en el uso de antibióticos y uso de antibióticos terapéuticos y profilácticos (situaciones clínicas médicas y odontológicas en las que contempla su uso, antibióticos de elección y dosis utilizadas).

En cuanto a su forma, para el cuestionario se consideraron preguntas de carácter uni- y multi-dimensional y cerradas (alternativas de respuesta delimitada). Se organizaron de tal forma que se comenzó con preguntas de carácter general y simples de responder y no se emplearon palabras que indujeran una respuesta estereotipada o que obligasen a hacer esfuerzos de memoria.

Para facilitar la respuesta del cuestionario de auto-reporte y reducir el tiempo que toma responderla, se aplicaron dos estrategias de organización. Primero, se empleó la condicionalidad de algunas respuestas para limitar las alternativas desplegadas en las siguientes preguntas. Segundo, en lugar de organizar linealmente las preguntas y sus alternativas de respuesta, se diseñaron matrices, en las cuáles, la combinación de lo descrito en las filas y columnas, constituye una pregunta del cuestionario. Para asegurar la respuesta a la mayoría de las preguntas y evitar que las últimas quedasen sin respuesta se diseñaron dos formas de presentación del cuestionario de auto-reporte: en una, la sección de prescripción de antibióticos terapéuticos se presenta primero y la profiláctica después y, en la otra forma, sucede a la inversa.

\section{Pilotaje del cuestionario de auto-reporte}

Se realizó con el fin de probar la pertinencia, eficacia y condiciones de aplicabilidad del instrumento creado ${ }^{(18)}$ y se efectuó vía electrónica por medio de la plataforma digital, SurveyMonkey. Se envió el cuestionario a través de una invitación por correo electrónico y las respuestas fueron recopiladas en forma anónima. Las respuestas se capturaron entre abril y mayo del 2019. El tamaño muestral fue calculado para un tamaño de universo infinito, error máximo aceptable de un $5 \%$, porcentaje supuesto de varianza del $50 \%$ y con un nivel de confianza del $95 \%{ }^{(18,19)}$. Según esto, el número mínimo de respuestas debía ser 384. Los odontólogos participantes fueron reclutados por conveniencia. Correspondió a una muestra heterogénea, incluyendo odontólogos generales y especialistas que trabajasen en el sector privado, público y/o universitario. Estos debían ser odontólogos chilenos o extranjeros (legalmente validados para su ejercicio profesional en nuestro país) y que estuviesen ejerciendo en Chile.

Para el desarrollo de la investigación se contó con el Auspicio del Ministerio de Salud de Chile, concedido por Resolución Exenta número 528 en Marzo del 2019.

\section{Consideraciones éticas}

Este proyecto fue aprobado por el Comité Ético-Científico de la Universidad de los Andes (Folio: CEC201834).

\section{RESULTADOS}

\section{Construcción del cuestionario de auto-reporte.}

El borrador de cuestionario inicial fue elaborado por los autores y la participación de 3 expertos. Estas reuniones tuvieron lugar entre octubre y noviembre de 2018, efectuándose las modificaciones al cuestionario sugeridos por estos. Los cambios fueron en relación a forma de presentación del cuestionario, redacción de las preguntas, dosis de antibióticos y cuadros clínicos a preguntar. El primer borrador consideró un total de 91 preguntas. Luego, entre diciembre 2018 y marzo 2019, se pidió la participación de informantes claves que permitieron calcular la validez de contenido del cuestionario por medio del Content Validity Ratio (CVR) y el Content Validity Index (CVI). Como informantes claves se contó con 8 participantes: 1 odontopediatra, 1 rehabilitador oral, 2 endodoncistas, 2 implantólogos y 2 periodoncistas, por lo que la mayor cantidad de preguntas debía tener un CVR mínimo de $0.75^{(16)}$

De las 91 preguntas originales, un $68,13 \%$ (62 preguntas) tuvieron un CVR igual a 1 ; un $18,68 \%$ (17 preguntas) tuvieron un CVR igual a 0,75 ; un $4,4 \%$ (4 preguntas) tuvieron un CVR igual a 0,5 ; un $3,3 \%$ ( 3 preguntas) tuvieron un CVR igual a 0,25 y un $5,49 \%$ (5 preguntas) tuvieron un CVR igual a 0 . De estas últimas 5 , hubo dos preguntas que, a pesar de su bajo CVR, los 
autores decidieron mantener. Estas fueron: en qué situaciones indica o ha indicado antibióticos (expectativas del paciente de recibir antibióticos, presión por poco tiempo y sobrecarga de trabajo, diagnóstico incierto, entre otros) y apreciación del cuestionario por el odontólogo. Las tres preguntas que fueron eliminadas, fueron consideradas como "no esenciales" y correspondían a preguntas sobre el sexo del encuestado, si el odontólogo solía derivar a diferentes especialidades o si solicitaba examen microbiológico al indicar antibióticos. La validez de contenido del cuestionario, medido por el CVI fue de 0,88 siendo aceptado según lo recomendado(16,17).

El proceso de diseño y validación mencionado resultó en un cuestionario de auto-reporte, conformado por 88 preguntas separadas en seis bloques: (A) 2 preguntas en relación a criterios de inclusión al estudio y consentimiento informado, (B) 7 preguntas de caracterización demográfica, (C) 4 preguntas sobre indicación general de antibióticos y cursos de actualización, (D) 41 preguntas sobre antibióticos terapéuticos, (E) 33 preguntas sobre antibióticos profilácticos y $(F) 1$ pregunta de apreciación del cuestionario al término del mismo.

\section{Pilotaje}

El cuestionario de auto-reporte fue enviado a 3209 correos electrónicos, obtenidos de base de datos pertenecientes a la Facultad de Odontología de la Universidad de los Andes y al registro nacional del Colegio de Cirujano Dentistas de Chile, entre el 11 de abril del 2019 hasta el 31 de mayo del 2019. De los 3209 correos, 284 no llegaron a destinatario, principalmente por presentar errores en la dirección de correo electrónico. Se recibieron un total de 577 respuestas (porcentaje de respuestas: 19,7\%). Un 67\% lo entregó completo (100\% de las preguntas) y un $72 \%$ (418 odontólogos) contestó al menos el $75 \%$ de las preguntas. Para efectos del análisis descriptivo de los hábitos de prescripción de antibióticos (Ver Tabla 1) se incluyeron en el análisis los 418 cuestionarios en que los odontólogos contestaron al menos un $75 \%$ de los ítems (Fowler (20)). Se descartaron 159 respuestas: 12 por no ser odontólogo o no tener su título legalmente validado en Chile, 3 por no estar de acuerdo con el consentimiento informado y 39 por no responder ninguna pregunta. La edad promedio de las 418 respuestas analizadas fue de 37,8 años, el $51,67 \%$ de las respuestas fueron de la Región Metropolitana. El $43,54 \%$ señaló tener mas de 10 años de experiencia laboral y el $63,88 \%$ indica realizar odontología general en su práctica diaria. Un $60,9 \%$ declaró trabajar en forma privada, un $26,32 \%$ en un consultorio de salud y un $26,08 \%$ en un centro odontológico. Un $50 \%$ de los odontólogos que respondieron el cuestionario declaró tener estudios de post-título. El $100 \%$ de odontólogos declaró indicar antibióticos de forma habitual, el $81 \%$ de ellos a entre $1 \%$ y $25 \%$ de sus pacientes el mes anterior al cuestionario y $8,6 \%$ del total a entre el $25 \%$ y $50 \%$ de sus pacientes. Amoxicilina es el antibiótico terapéutico y profiláctico de elección para la mayoría de las condiciones clínicas descritas, seguido por la combinación de Amoxicilina/ Acido Clavulánico y Amoxicilina/Metronidazol. Azitromicina es el antibiótico terapéutico y profiláctico de elección en pacientes alérgicos a las penicilinas (67,96\% y $61,74 \%$ respectivamente). Frente a un mismo cuadro clínico se detectó una importante variabilidad en la elección del antibiótico así como en las dosis y el tiempo de duración indicada para la ingesta. Un porcentaje de odontólogos, aunque menor, prescribe antibióticos frente a cuadros virales, como herpes simple o cuadros inflamatorios como gingivitis. El tiempo promedio que le tomó a los odontólogos responder el cuestionario fue de 15 minutos. Al momento de valorar el cuestionario, la mayoría $(84,31 \%)$ lo consideró excelente o muy bueno.

\section{DISCUSIÓN}

La venta y uso de antibióticos en Chile, mostró un aumento progresivo en el consumo entre los años 1988-1997(21). En Septiembre de 1999 el Ministerio de Salud de Chile implementó la medida de "Uso Racional de Antibióticos" , restringiendo su dispensación en farmacias sólo con receta médica (Ordinario 4C/5015 del 30.09.1999) logrando un impacto en la disminución de la venta de estos medicamentos. Bavestrello y cols ${ }^{(22)}$ evaluó el impacto de estas medidas regulatorias y reportó una disminución relevante en las ventas de antibióticos entre los años 1999-2000. Sin embargo, aparentemente, estas medidas regulatorias no logran un impacto de largo plazo. Datos reportados por el Instituto de Salud Pública en Noviembre de 2019, indicaba que entre los años 2014 al 2019 se produjo un aumento en el consumo de un $17,4 \%$ para Amoxicilina; 34\% para Azitromicina; $22,9 \%$ para Amoxicilina / Ac.Clavulánico, entre otros, con niveles similares a los observados 15 años antes ${ }^{(23)}$

A pesar de que a nivel mundial los odontólogos son responsables de un 7 a un $10 \%$ de las prescripciones de antibióticos ${ }^{(5-7)}$ y existen varios estudios sobre este tema ${ }^{(8-12)}$, en Chile no se había creado a la fecha ningún instrumento que permitiese evaluar la prescripción de antibióticos por los odontólogos en nuestro país. El acto de medir es un componente esencial en la investigación científica, ya sea en las ciencias naturales, sociales o de la salud ${ }^{(14)}$ y es abordable mediante la creación de un instrumento
Tabla 1: Distribución demográfica odontólogos encuestados.

\begin{tabular}{l|l|l}
\hline \multicolumn{1}{c|}{ Variable } & Frecuencia & $\begin{array}{c}\text { Porcentaje (\%) } \\
\text { (N=418) }\end{array}$ \\
Años de ejercicio profesional & \multicolumn{1}{l}{} \\
\hline 0-2 años & 17 & 4,07 \\
\hline 3-6 años & 143 & 34,21 \\
\hline 7-9 años & 76 & 18,18 \\
\hline Igual o más de 10 años & 182 & 43,54 \\
\hline Nivel máximo de estudio & \multicolumn{2}{|l}{} \\
\hline Pregrado & 34 & 8,13 \\
\hline Postítulo & 209 & 50 \\
\hline Magister & 68 & 16,27 \\
\hline Doctorado & 4 & 0,96 \\
\hline Otros (diplomados, etc.) & 103 & 24,64 \\
\hline Lugar de trabajo* & \multicolumn{2}{|l}{} \\
\hline $\begin{array}{l}\text { Clínica Universitaria } \\
\text { Docente-asistencial }\end{array}$ & 94 & 22,49 \\
\hline Hospital y/o Clínica & 90 & 21,53 \\
\hline Consulta Privada & 252 & 60,29 \\
\hline Centro Odontológico & 109 & 26,08 \\
\hline Consultorio & 110 & 26,32 \\
\hline Fuerzas Armadas & 16 & 3,83 \\
\hline Otra & 12 & 2,87 \\
\hline
\end{tabular}

Especialidad a la que se refiere su nivel máximo de estudio**

\begin{tabular}{l|l|l}
\hline Cirugía Oral & 32 & 7,66 \\
\hline Cirugía Maxilofacial & 18 & 4,31 \\
\hline Endodoncia & 62 & 14,83 \\
\hline Implantología & 66 & 15,79 \\
\hline Odontología General & 69 & 16,51 \\
\hline Odontopediatría & 41 & 9,81 \\
\hline Ortodoncia & 36 & 8,61 \\
\hline Patología Oral & 13 & 3,11 \\
\hline Periodoncia & 70 & 16,75 \\
\hline Radiología Oral & 13 & 3,11 \\
\hline Rehabilitación Oral & 61 & 14,59 \\
\hline Trastornos & 17 & 4,07 \\
\hline Oemporomandibulares & 47 & 11,24 \\
\hline
\end{tabular}

*La frecuencia corresponde a lo que reportan los encuestados, considerando que podían marcar más de una alternativa de lugar de trabajo. El porcentaje en cambio, es calculado a partir del total de 418 sujetos.

** La frecuencia corresponde a lo que reportan los encuestados, considerando que podían marcar más de una especialidad. El porcentaje en cambio, es calculado a partir del total de 418 sujetos.

adecuado que permita registrar de manera confiable, válida y objetiva datos observables ${ }^{(18)}$

Los cuestionarios de auto-reporte son, tal vez, los instrumentos más utilizados para recolectar datos. Estos consisten en un conjunto de preguntas respecto a una o más variables y en términos generales, recogen, cuantifican, universalizan y comparan información ${ }^{(18)}$. Siguiendo a Streiner y colegas ${ }^{(14)}$, para la creación de las preguntas del presente cuestionario de auto-reporte se utilizaron, investigaciones previas, opinión de expertos y entrevistas a informantes claves. Metodologías similares al del presente estudio se han realizado en países como el Reino Unido $(6,8,12,24)$, Estados Unidos $^{(7)}$, Noruega ${ }^{(9-11)}$, Alemania ${ }^{(25)}$ y Brasil(13), entre otros. Estos trabajos permitieron identificar una serie de falencias en la indicación, posología, intervalos y duración de la ingesta.

En relación a la validación del instrumento, una declaración de validez 
aparente y de contenido, basada en alguna revisión por un panel de expertos o informantes claves, es un requisito previo mínimo para la aceptación de un instrumento ${ }^{(14)}$. De esta manera, nuestro estudio considera la medición del Content Validity Ratio (CVR) y Content Validity Index (CVI)(16,17) donde la mayoría de las preguntas se consideraron adecuadas, dado que los valores superan el punto de corte mínimo de 0.75 .

La tasa de respuestas para el cuestionario de auto-reporte aplicado fue de $19,7 \%$. La mayoría de los estudios similares a este tienen una tasa de respuesta superior. Así por ejemplo en Reino Unido, Palmer y cols ${ }^{(8)}$ reporta un $60 \%$ de respuestas; en Noruega, Preus y cols ${ }^{(10)}$ señala un $78 \%$ y Choudhury y cols ${ }^{(24)}$ un $73 \%$. Una tasa de respuestas más cercana a nuestros resultados obtuvo Falkenstein y cols $^{(25)}$ que reporta un $29,1 \%$. Estas diferencias probablemente se deben al diferente método de entrega del cuestionario a los odontólogos que, en los estudios mencionados, se realizó vía correo certificado y no vía correo electrónico. Los beneficios de utilizar una cuestionario online y como auto-reporte para este estudio, fue su reducido costo de aplicación y su rapidez y facilidad en la colección de datos. Además, se redujo el posible error humano en la codificación y traspaso de las respuestas y no hubo limitación geográfica ni de horario para la obtención de éstas. El tiempo promedio de respuesta del cuestionario fue de 15 minutos, que consideramos adecuado. Esto se debe al diseño aplicado, con un número de preguntas acotado, el despliegue lógico de estas y su fácil lectura.

Relativo a prescripción, Amoxicilina es el antibiótico de elección en casi todas las situaciones clínicas señaladas en el cuestionario de auto-reporte. Esta situación es similar a lo señalado por los otros estudios mencionados. La principal diferencia se establece en la indicación del antibiótico alternativo en caso de alergia a Penicilinas. En nuestro estudio, Azitromicina fue el más indicado en situaciones de tratamiento y profilaxis a diferencia de investigaciones en Reino Unido ${ }^{(12)}$ en que el fármaco más indicado fue Clindamicina seguido por Eritromicina. Una probable explicación a esto radica en la indicación de posología de la Azitromicina que ayuda en el cumplimiento de la ingesta y en que es un fármaco relativamente reciente en el mercado.

Este estudio es el primer intento realizado en Chile por crear un instrumento de recolección de información relativo a la indicación y uso de antibióticos por los odontólogos y es parte de una línea de investigación orientada a conocer los hábitos de prescripción de nuestra profesión. Entre sus limitaciones más importantes está la baja tasa de respuesta, que es posible atribuir a la falta de tiempo, desinterés o bien desconfianza para responder cuestionarios a través de plataformas digitales.

\section{CONCLUSIONES}

El instrumento de recolección de información es válido para ser usado por odontólogos en Chile. El cuestionario de auto-reporte creado permitió levantar información preliminar sobre la prescripción de antibióticos por odontólogos en Chile.

\section{RELEVANCIA CLÍNICA}

Justificación científica para el estudio: El uso racional de medicamentos es prioridad para el Ministerio de Salud. No se conocen los hábitos de prescripción de antibióticos por parte de los dentistas en Chile.

Resultados principales: Se diseña y valida un cuestionario de autoreporte que permite levantar información de los hábitos de prescripción profiláctica y terapéutica de los odontólogos en Chile.

Consecuencias prácticas: Los datos obtenidos a partir de la aplicación del instrumento diseñado son válidos y representan a la comunidad odontológica ejerciendo en Chile.

\section{FINANCIAMIENTO}

La investigación se financió con recursos propios de la Asignatura de Periodoncia de la Facultad de Odontología de la Universidad de los Andes.

\section{AGRADECIMIENTOS}

Los autores están agradecidos de la generosidad del Dr. Hans Preus al compartir con ellos su experiencia. También agradecemos la colaboración de Bárbara Hanisch, Psicóloga Educacional y doctorada en Economía, del profesor Claudio Romero, magister en Atención Farmacéutica Hospitalaria, del Dr. Francisco Muñoz, Cirujano Maxilofacial y de todos los informantes claves que participaron en la validación del cuestionario de auto-reporte.

\section{CONFLICTOS DE INTERÉS}

Los autores declaran no tener conflictos de interés.

\section{Bibliografía}

1. Ministerio de Salud. Gobierno de Chile. Uso racional de medicamentos. [consultado 18/092017]. Disponible en: http://web.minsal.cl/medicamentos_uso_racional/ 2. Ministerio de Salud. Gobierno de Chile. Manual selección de medicamentos. [consultado 18/092017]. Disponible en: http://web.minsal.cl/sites/default/files/ files/Manual\%20Seleccin $\% 20$ de $\% 20$ Medicamentos $\% 20$ FInal $\% 20$ con $\% 20$ Dise\%C3\%B1io.pdf

3. Ministerio de Salud. Gobierno de Chile. Guía para las buenas prácticas de prescripción. [consultado 18/09/2017]. Disponible en: http://web.minsal.cl/wpcontent/uploads/2015/09/2CD_GUIA-PARA-LAS-BUENAS.pdf

4. World Health Organization, editor. The Rational use of drugs: report of the conference of experts, Nairobi, 25-29 November 1985. Geneva : Albany, NY: World Health Organization ; WHO Publications Center USA [distributor]; 1987. 329 p. 5. Cope G, Cope A. Antibiotic resistance and how to act on it. Dent Nurs [Internet]. 2013 Dec [cited 27/12/2019];9(12):706-9. Available from: http://www. magonlinelibrary.com/doi/10.12968/denn.2013.9.12.706

6. Chate RAC, White S, Hale LRO, Howat AP, Bottomley J, Barnet-Lamb J, et al. The impact of clinical audit on antibiotic prescribing in general dental practice. Br Den J [Internet]. 2006 Nov 25 [cited 22/04/2017];201(10):635-41. Available from: http:// www.nature.com/doifinder/10.1038/sj.bdj.4814261

7. Fluent MT, Jacobsen PL, Hicks LA. Considerations for responsible antibiotic use in dentistry. J Am Dent Assoc [Internet]. 2016 [cited 2017 Apr 22];147(8):683-686 Available from: http://jada.ada.org/article/S0002-8177(16)30409-3/fulltext?rss=yes 8. Palmer NAO, Pealing R, Ireland RS, Martin MV. Therapeutics: a study of therapeutic antibiotic prescribing in National Health Service general dental practice in England. Br Dent J [Internet]. 2000 [cited 22/04/2017];188(10):554-558. Available from: http://www.nature.com/bdj/journal/v188/n10/abs/4800538a.html

9. Demirbas F, Gjermo PE, Preus HR. Antibiotic prescribing practices among Norwegian dentists. Acta Odontol Scand [Internet]. 2006 Jan [cited 30/102017];64(6):355-9 Available from: http://www.tandfonline.com/doi/full/10.1080/00016350600844394

10. Preus HR, Fredriksen KW, Vogsland AE, Sandvik L, Grytten JI. Antibi-oticprescribing habits among Norwegian dentists: a survey over 25 years (1990-2015). Eur J Oral Sci [Internet]. 2017 Aug [cited 30/102017];125(4):280-7. Available from: http://doi.wiley.com/10.1111/eos.12360

11. Preus HR, Albandar JM, Gjermo P. Antibiotic prescribing practices among Norwegian dentists. Eur J Oral Sci. 1992;100(4):232-235

12. Palmer NAO, Pealing R, Ireland RS, Martin MV. A study of prophylac-tic antibiotic prescribing in National Health Service general dental practice in Eng-land. Br Dent J [Internet]. $2000 \mathrm{Jul}$ [cited 2019 Dec 27];189(1):43-6. Available from: http://www. nature.com/articles/4800597
13. Monteiro AV, Ribeiro FV, Casarin RCV, Cirano FR, Pimentel SP, Casati MZ. Evaluation of the use of systemic antimicrobial agents by professionals for the treatment of periodontal diseases. Braz J Oral Sci [Internet]. 2013 Dec [cited 11/06/2020];12(4):285-91. Available from: http://www.scielo.br/scielo.php?script=sci_ arttext\&pid=S1677-32252013000400003\&lng=en\&nrm=iso\&tlng=en

14. Streiner DL, Norman GR, Cairney J. Health measurement scales: A practical guide to their development and use. Oxford University Press; 2015. 415 p.

15. Haynes S, Richard D, Kubany, E. Content validity in psychological as-sessment: A functional approach to concepts and methods. Psychol Assess. 1995 Sep 1:7:238-47. 16. Lawshe $\mathrm{CH}$. A Quantitative approach to content validity 1. Pers Psy-chol [Internet]. 1975 Dec 1;28(4):563-75. Available from: https://onlinelibrary.wiley.com/doi/ abs/10.1111/j.1744-6570.1975.tb01393.x

17. Gilbert G, Prion S. Making sense of methods and measurement: Law-she's content validity index. Clin Simul Nurs. 2016 Dec 1;12:530-1.

18. Roberto Hernández Sampieri, Carlos Fernández Collado, Pilar Baptista Lucio. Metodología de la investigación. 5a. ed. McGraw-Hill Interamericana; 2010.

19. Investigación de mercado y opinión pública [Internet]. Cadem. [con-sultado 22/03/2019]. Disponible en: http://www.cadem.cl

20. Floyd J. Fowler. Survey research methods. 5a ed. SAGE publications; 2014

21. Bavestrello F L, Cabello M Á. Consumo comunitario de antimicrobianos en Chile, 2000-2008. Rev Chil Infectol [Internet]. 2011 Apr [consultado 11/06/2020];28(2):10712. Disponible en: https://scielo.conicyt.cl/scielo.php?script=sci_abstract\&pid=S0716 10182011000200001\&lng=es\&nrm=iso\&tlng=es

22. Bavestrello F L, Cabello M A, Casanova Z D. Impacto de medidas regulatorias en la tendencia de consumo comunitario de antibióticos en Chile. Rev Médica Chile [Internet]. 2002 Nov [consultado22/04/2017];130(11):1265-72. Disponible en: http://www.scielo.cl/scielo. php?script=sci_abstract\&pid=S0034-98872002001100009\&lng=es\&nm=iso\&tlng=es 23. ISP informa sobre la resistencia a los antimicrobianos y antibióticos más vendidos en el país [Internet]. www.ipsuss.cl. [consultado 2020 Jun 11]. Disponible en: http:// www.ipsuss.cl/ipsuss/site/artic/20191129/pags/20191129121331.htm

24. Choudhury M, Needleman I, Gillam D, Moles DR. Systemic and local antimicrobial use in periodontal therapy in England and Wales. J Clin Periodontol [Internet]. 2001 [cited 11/06/2020];28(9):833-9. Available from: https://onlinelibrary.wiley.com/doi/ abs/10.1034/j.1600-051x.2001.028009833.x

25. Falkenstein S, Stein JM, Henne K, Conrads G. Trends in antibiotic use and microbial diagnostics in periodontal treatment: comparing surveys of German dentists in a tenyear period. Clin Oral Investig [Internet]. 2016 Nov 1 [cited 11/06/2020];20(8):2203-10. Available from: https://doi.org/10.1007/s00784-016-1722-6. 